\title{
Merry Christmas!
}

Im Deutsch-Amerikanischen Institut in Heidelberg habe ich traditionell die US-Wahlnacht verbracht und war verblüfft wie viele Mitbürger: Just am 9. November, dem Tag als in Deutschland einst die Mauer fiel, siegt Donald Trump und wird der 45. amerikanische Präsident der USA, ein Mann, der im Wahlkampf wiederholt für eine Mauer als Mittel der Zuwanderungsbegrenzung zwischen USA und Mexiko warb. Für ihn kommt Amerika zuerst, Europa muss seine Interessen neu definieren und ausrichten. In Amerika dominiert der Mainstream, manche sagen auch die „Straße“, und somit die Existenzprobleme des amerikanischen Mittelstandes, der sich seit Jahren im permanenten Sinkflug befindet. Ausbau des Umweltschutzes, Ressourcen-schonendes Wirtschaften, erneuerbare Energien, manche dieser Themen werden unter Donald Trump neu definiert und umgesetzt werden, manche Kommentatoren rechnen gar mit einer Renaissance fossiler Energieträger. TTIP-Gegner können sich freuen. Trump ist ein Gegner des Vertragswerks. Mit der Wahl Donald Trumps endet die Zeit nach dem Zweiten Weltkrieg endgültig.

Dazu bemerken wir zum Teil doch erstaunliche Parallelen bei uns: zunächst in Europa das Erstarken rechtsnationalistischer und -populistischer Parteien, auch hier immer mehr Arbeit für immer weniger Geld, die beginnende Verdrängung von Arbeitsplätzen durch Computer und Roboter, dazu viele ungelöste Probleme in fast allen Politikbereichen durch fehlenden politischen Konsens und Weitblick! Flickschusterei nicht nur in der Gesund-

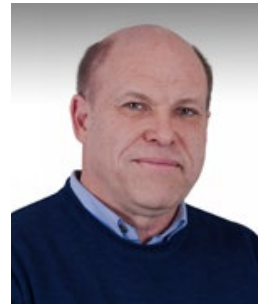

Dr. Matthias Herbst

Generalsekretär ADK e. V.

heitspolitik, sondern überall. Europa muss selbstständig werden oder wird bedeutungslos. Die von oben propagierte Globalisierung führt zu neuen Flüchtlingsströmen, deren Bewältigung ist umstritten und teuer. Die viel größere Krise ist aber die Erosion des Bankensektors und des Vertrauens der Bevölkerung in den Euro durch die Negativzinspolitik der europäischen Zentralbank.

Wenn selbst der Gesundheitsfond der Krankenkassen Negativzinsen in Millionenhöhe berappen muss, kann etwas mit unserer Politik nicht stimmen. Es ist die schleichende Geldentwertung und der damit verbundene Kaufkraftverlust, der zum Beispiel in der Gebührenordnung der Ärzte seit 1983 für eine Entwertung des ärztlichen Honorars in einer Höhe von fast $75 \%$ geführt hat. Für die Ärzte heißt es in immer weniger Zeit immer mehr Leistungen erbringen zu müssen. 
Da sollte bei einer neuen Gebührenordnung für die Ärzte natürlich ein entsprechendes Plus im Honorar erscheinen. Wenn nun der Verhandlungsführer der Ärzte selbst erklärt, dass ein Mehr von 6,4\% insgesamt alles sei, was er für uns verhandeln könne, muss man aufhorchen. Die Versäumnisse der Vergangenheit rächen sich jetzt. Es stellt sich die Frage nach der richtigen Strategie der Bundesärztekammer in den Honorarverhandlungen. Offensichtlich sind der fehlende Konsens und die Geheimhaltung die Hauptprobleme.

Anstatt zunächst einmal mit den Kassen eine erste lineare Erhöhung der Gebührenordnung zu verhandeln, öffnet man sofort die Büchse der Pandora und versucht zunächst die Bewertung der einzelnen Leistungen den aktuellen Kostenstrukturen anzupassen, um am Ende ein Maximum von 6,4\% Honorarplus herauszuholen. Gegnern dieses Vorgehens wird zudem vom Verhandlungsführer der Ärzteschaft das Auswandern nahegelegt. Ein Arzt müsse doch auch mit einem Platz in der zweiten Klasse im ICE zufrieden sein und auch die Wahl des Autos könne bescheidener ausfallen.

Gleichzeitig wird durch diese Art der Verhandlungsführung viel Misstrauen erregt, da auch niemand weiß, was zum Beispiel mit den individuellen Gesundheitsleistungen und deren Abrechnung geschehen soll. Es ist also an alle Beteiligten $\mathrm{zu}$ appellieren, nicht noch lange herumzutaktieren, sondern die Karten auf den Tisch zu legen. Gibt es keinen erkennbaren Willen der Krankenkassen und der Politik, den Ärzten endlich ein leistungsgerechtes Honorar zuzugestehen, sollte man die Verhandlungen eher abbrechen. Es muss vor allen Dingen überlegt werden, ob nicht auch den Patienten in Zukunft Mitwirkungspflichten auferlegt werden, zum Beispiel wenn man als Arzt erkennt, dass Empfehlungen des Lichtschutzes zur Vorbeugung von Hautkrebs in keiner Weise beachtet werden. Warum soll dann der Patient nicht eigene Anteile an seiner Behandlung übernehmen? Damit man da trifft, wo er am empfindlichsten ist - nämlich an seinem Portemonnaie. Auch könnte die Behandlung zu besonderen Wunsch-/Unzeiten in der Praxis neu taxiert werden, um den Behandlungsfluss besser zu steuern.

Im Wettbewerb mit den Vereinigten Staaten und in einer globalisierten Welt hilft nur die Effizienz. Diese muss sowohl die notwendige System-Bürokratie wie auch die Qualität der Leistungen erfassen und für ein sinnvolles, transparentes und verständliches Abrechnungsgeschehen sorgen, dass den Patienten zum direkten Partner des Arztes macht.

Die Politik muss wieder lernen, für Mehrheiten attraktiv zu sein und so Populisten keine Chance zu geben. Dies kann sie nur, indem sie sich für nachvollziehbare Leistung und Kosteneffizienz - auch im Gesundheitswesen - entscheidet. Zugleich muss ärztliche Leistung entsprechend belohnt werden!

Die Arbeitsgemeinschaft Ästhetische Dermatologie \& Kosmetologie (ADK) wird auch im neuen Jahr versuchen, hier hinter den Kulissen sinnvoll zu agieren. Der ADK-Vorstand wünscht seinen Mitgliedern und Freunden ein ruhiges und gesegnetes Weihnachtsfest und für 2017 alles Gute!

Denken Sie daran: Miteinander bringt für alle immer mehr und uns gemeinsam weiter! Wie immer herzlich

Ihr

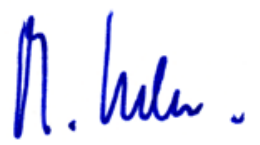

Dr. Matthias Herbst 